\title{
Treating scleroderma lung disease with B cell depletion
}

\author{
Constantina A Bounia ${ }^{1}$ and Stamatis-Nick C Liossis ${ }^{1,2 *}$ \\ ${ }^{1}$ Division of Rheumatology, Patras University Hospital, GR 26500 Rion, Patras, Greece \\ ${ }^{2}$ Department of Internal Medicine, University of Patras Medical School, GR 26500 Rion, Patras, Greece
}

\begin{abstract}
Systemic sclerosis $(\mathrm{SSc}$ ) is a rare, aggressive, systemic, autoimmune disease especially because of internal (vital) organ involvement, most commonly over the last decades of the lungs. Therefore, there is an increasing need for the rheumatologist to select an effective treatment in order to improve patients' prognosis.

Recent experimental data have established a role of the B cell in pathogenesis of the disease. B cell depletion, although not usually chosen as first-line treatment, has been investigated over the past decade in controlled and uncontrolled trials with satisfactory results mainly in skin involvement, but trials of B cell depletion in improving lung function do not lag behind in effectiveness compared to respective results in the skin. This review is referring to trials that have attributed $\mathrm{B}$ cell depletion an optimistic view of halting progression of interstitial lung disease in scleroderma.
\end{abstract}

\section{Introduction}

Systemic Sclerosis is a chronic, autoimmune disease affecting not only the skin but also internal organs. The scope of our review is to focus on lung involvement. Autoimmunity and vasculopathy are thought to end up in inflammation and fibrosis simultaneously, as manifested not only in the skin but also in internal organs [1].

Interstitial lung disease (ILD) occurs early especially in the diffuse type of SSc with varying progression. Pulmonary function tests (PFTs) may not be useful in early detection and screening of scleroderma ILD. Screening is based upon chest high resolution computed tomography images (HRCT) displaying 2 patterns: predominantly non-specific interstitial pneumonia (NSIP) and less frequently usual interstitial pneumonia (UIP). The extent of ILD in HRCT and the decline of PFTs, in particular of Forced Vital Capacity (FVC) and Diffusing lung capacity for carbon monoxide (DLCO) are prognostic parameters for poorer outcomes as the disease progresses $[2,3]$.

The inflammatory process, the fibrosis and the impaired pulmonary function are rather challenging to researchers $[4,5]$. As researchers discover new pathways more relevant to the progression of the disease, the selection of the appropriate treatment for SSc seems more attainable. Treatment options are few and most are not able to hinder the progression of SSc especially in the lung. Current and future perspectives on treatment of SSc have been promising [6,7]. B cell depletion has been gaining increasing interest in treating successfully enough patients with SSc ILD during the past 10 years [7-10]. Case reports and controlled or uncontrolled studies strengthen this assertion $[11,12]$.

Studies in experimental animals and in humans with SSc have implicated the semantic role of B cells in the pathogenesis of the disease $[5,13,14]$. Apart from the peripheral blood, B cell infiltrates are apparent in lung tissue from SSc patients as well [15].

\section{B cell depletion in experimental animal models}

Only a few animal models exist mimicking the SSc clinical phenotype. Tight skin mice (TSK) manifest skin fibrosis and display circulating autoantibodies against topoisomerase similarly to humans. Enhancement of CD19-mediated signaling was found in TSK B cells, while CD19 knockout TSK mice displayed improved skin fibrosis and reduced autoantibody production [16-19]. In addition, B cell depletion in newborn TSK mice reduced skin fibrosis, hypergammaglobulinemia and autoimmunity in contrast to old TSK mice [18]. Similarly, B cell activating factor (BAFF) which is essential for B cell survival, is elevated in the sera of TSK mice, while the administration of the anti-BAFF $\mathrm{mAb}$ belimumab ameliorated skin fibrosis and inhibited autoimmunity and production of inflammatory cytokines [19].

Another model of SSc is that of mice injected with bleomycin; such mice develop skin and lung fibrosis. CD19-mediated signaling is associated with pulmonary fibrosis in this model, while CD19 deficiency prevented lung fibrosis induced by bleomycin injection [20]. Graft Versus Host Disease (GVHD) is another clinical paradigm of nearly identical to SSc lung fibrosis. Allen et al. [21] studied B cells from patients with GVHD and found an increase of their numbers and metabolic state and a decrease of their apoptosis rates as well. The administration of exogenous BAFF amplified B cell size and further enhanced B cell activation and survival.

On the other hand, Le huu et al. [19] found that transplantation of Bregs from mice-donors who lack CD19 provoked more lung and skin fibrosis and GVHD severity in irradiated Balb-c mice whereas the transplantation from wild-type mice donors did not, thus implying a suppressive role of Bregs in SSc. Lafyatis et al. [15] found B cells both as lymphoid aggregates and as diffuse aggregates in pulmonary tissue of 11 patients with SSc-associated ILD; of these, 7/11 had a UIP and the rest 4/11 had a NSIP phenotype.

${ }^{\star}$ Correspondence to: Stamatis-Nick C. Liossis, MD, Dept of Internal Medicine, Patras University Hospital, 5th floor, GR 26500, Rion, Patras, Greece, E-mail: snliossis@med.upatras.gr

Received: September 12, 2020; Accepted: September 22, 2020; Published: September 25, 2020 


\section{Studies of $B$ cell depletion in humans}

\section{A) Controlled trials}

The first randomized controlled study of Rituximab (RTX) in SSc-ILD by Daoussis et al. [22] evaluated 8 patients receiving RTX as an add-on therapy to standard treatment, versus 6 patients receiving only conventional treatment. All patients had extensive ILD. They received RTX at 4 weekly pulses at a dosage of $375 \mathrm{mg} / \mathrm{m} 2$ ("lymphoma scheme"). A significant increase of FVC was recorded in the RTX group compared with baseline values (mean \pm S.D.: $68.13 \pm 19.69$ vs. $75.63 \pm$ 19.73 , at baseline vs 1 year, respectively, $\mathrm{p}=0.0018$ ) and of the DLCO as well (mean \pm S.D.: $52.25 \pm 20.71$ vs. $62 \pm 23.21$, at baseline vs. 1 year, respectively, $\mathrm{p}=0.017$ ). The conventional treatment group had no significant changes of FVC and DLCO compared to the RTX group. Finally, the chest HRCT scores defining the extent of lung involvement remained stable at 24 weeks in the RTX group, while they worsened slightly in the control group.

Jordan et al. [23] matched 25 RTX-treated patients with 25 control patients without RTX treatment from the EUSTAR database for a 6-month period. RTX-treated patients displayed a stable FVC (60.6 \pm 2.4 vs $61.3 \pm 4.1 \%$; $=0.5)$ and a significantly increased DLCO (41.1 \pm 2.8 vs $44.8 \pm 2.7 \%$; $\mathrm{p}=0.03$ ) compared to baseline. A direct comparison between the 2 groups of patients (RTX-treated and RTX-untreated) also revealed significant changes in both the percentages of predicted FVC $(0.4 \pm 4.4$ vs $-7.7 \pm 3.6 ; p=0.02)$ and the absolute FVC change $(0.8 \pm 2.2$ vs $-4.8 \pm 1.7 ; \mathrm{p}=0.01)$. On the contrary, no significant changes of DLCO between two groups were reported ( $3.7 \pm 1.4$ vs $6.2 \pm 6.2$; $\mathrm{p}=0.9$ ).

In the multicenter (7 rheumatology clinics) open label study of B cell depletion from Greece, Daoussis et al. [24] aimed to assess longterm efficacy and safety of RTX in the ILD of patients with SSc. Fiftyone patients received RTX on top of standard treatment and 33 received standard treatment alone; patients were followed up for a median time of 4 years. During the first 2 years, a significant increase of FVC was shown in the RTX group (mean \pm SD of FVC: $80.6 \pm 21.21$ vs. $86.90 \pm$ 20.56 at baseline vs. 2 years, respectively, $p=0.041$ ). On the contrary, the patients on standard treatment alone, displayed a stabilization of their FVC. In addition, patients that completed 7 years of RTX treatment $(n=5)$ had numerically but not statistically higher FVC compared to baseline (mean \pm SD of FVC : $91.60 \pm 14.81, \mathrm{p}=0.158$ ), while the patients $(n=9)$ on standard treatment had FVC deterioration $(p<0.01)$. However, a direct comparison between the two groups disclosed a significant benefit for the RTX group ( $p=0.013$ ). Treatment with RTX did not alter DLCO throughout the 7-yr period, while patients on standard treatment alone, displayed a significant reduction of DLCO $(\mathrm{p}=0.004)$. It is worthwhile to mention that a temporary cessation of therapy in 6 patients with SSc was associated with a significant decline of FVC; restarting RTX in a subsequent increase of FVC in 3 only patients.

Boonstra $\mathrm{M}$ et al. [25] studied patients with early SSc. Half of them were treated with RTX $1000 \mathrm{mg}$ biweekly ("RA scheme") and the rest of them with placebo treatment over a period of 2 years. All patients had been taking immunosuppressive therapy previously. The study disclosed that after 2 years FVC and the extent of lung involvement slightly improved with RTX, but this difference was insignificant when compared to the placebo group. FVC (placebo: -1.4 vs RTX: +4 , $\mathrm{p}=0.65$ ), DLCO (placebo: -2.2 , RTX: $-6.0, \mathrm{p}=0.77$ ). Analysis of HRCT data according to criteria set by Goh showed a mean change in the percentage of affected lung tissue between baseline and 12 months of $-1.6 \%$ for the RTX and $+2.8 \%$ for the placebo treated groups $(\mathrm{p}=0.28)$.

Thiebeau et al. [26] compared 13 patients with SSc-ILD who received
RTX after already having being treated with immunosuppressants with 26 patients with SSc-ILD that did not receive RTX for a 2-year period. FVC was practically unchanged at 12 months; $72 \%$ at baseline and $85 \%$ at month $12(\mathrm{p}=0.6)$, as was the DLCO; $40 \%$ at baseline $49 \%$ at month $12(\mathrm{p}=0.9)$. During the second year of follow-up, 7 patients in the RTX group improved their FVC gain by 12 points. In contrast, 14 non-RTX treated patients worsened their FVC loss by 1.5. A direct comparison between the 2 groups disclosed a statistically significant improvement of RTX-treated patients $(p=0.003)$. DLCO also improved in the RTX group with a gain of 4 , while it worsened with a loss of -4.5 in the nonRTX group. Once again, the comparison between the 2 treatment arms showed a superiority of RTX-treated patients vs. the non-RTX-treated in improving DLCO $(\mathrm{p}=0.03)$. The same authors also analyzed a total of 42 patients ( 35 from the literature and 7 from their own series). This analysis revealed an increase of FVC from $71 \%$ at baseline to $84 \%$ at 12 months $(\mathrm{p}=0.0006)$ and an increase of DLCO from $58 \%$ at baseline to $64 \%$ at 12 months $(\mathrm{p}=0.02)$ in patients treated with RTX.

The EUSTAR study evaluated 146 out of 254 patients with SSc ILD who received RTX plus standard treatment vs. patients only on standard treatment for a 2-year follow-up period [27]. FVC remained stable during the follow-up in the RTX group (76.3 to 77.7) and in the standard treatment group as well (79.1 to 80.7). DLCO also remained stable both in the RTX treated patients (54.4 to 55.5) and in the standardof-care treated patients (55.6 to 54.7). Despite no significant changes seen in the lung function of the RTX group, these patients reduced the dosage of daily steroids earlier and displayed an acceptable tolerance on drug administration. Although the number of patients was large enough, their lung involvement differed in extent and, perhaps more importantly, in chronicity and the protocol of RTX administration was also different among the participating medical centers. Therefore, a future trial could be designed in order to diminish such confounding heterogeneity that might potentially mask treatment-induced changes of outcomes.

In a recent open-label, randomized, controlled trial Sircar et al. [28] compared head-to-head RTX vs. monthly cyclophosphamide (CYC) treatment. Sixty patients with early, treatment-naïve, anti-Scl70(+), diffuse SSc with ILD were enrolled to receive RTX or CYC. Patients in the CYC treatment arm received $500 \mathrm{mg} / \mathrm{m} 2$ CYC IV pulses every 4 weeks for 24 weeks $[6,29]$. Patients in the RTX group received RTX as in the RA scheme. They authors found the RTX group to have improved FVC at the end of 6 months when compared to baseline values (RTX group: $61.3 \%$ to $67.5 \%$ ) while the CYC group did not (CYC: $59.3 \%$ to $58.1 \%)$. Not only the efficacy but also the safety demonstrated in this trial argues that RTX may be considered as a first-line therapy versus CYC. However, the current standard-of-care treatment is mycophenolate mofetil (MMF) and not CYC. Another study from Japan also favors RTX $(n=9)$ versus CYC $(n=30)$ in the treatment of patients with SScILD [30].

Evaluation of efficacy and safety of RTX plus MMF in patients with ILDs (EvER-ILD) is an ongoing study (7). A broad range of patients with non-responding resistant ILD was recruited to receive different therapies and lung function was evaluated in 6 months. One group received RTX (RA scheme) plus MMF. The other group received one placebo infusion plus MMF for 6 months. Controlled trials are collectively depicted in Table 1.

\section{B) Uncontrolled studies}

The study by Lafyatis et al. [31], who treated 15 patients with early SSc with RTX only (RA scheme) without concomitant disease modifying anti-rheumatic (DMARD) therapy did not find a clear 
beneficial effect on skin fibrosis and pulmonary function at 6 and 12 months of follow-up. However, patients with severe lung disease were excluded. The average FVC and DLCO showed no significant differences at 6-months $(92.7 \%$ and $77.9 \%$ predicted, respectively) compared to the baseline $(89.2 \%$ and $79.7 \%$ predicted, respectively). In addition, none of the patients showed new or progressive pulmonary disease by HRCT. It might be noticed however that patients included had near-normal respiratory function limiting thus the potential results of any treatment.

Daoussis et al. [8] showed a beneficial effect of RTX (lymphoma scheme) also after 2 years of treatment in our initial cohort of 8 patients from the study previously analyzing then at 1 year. An outstanding increase in FVC (mean \pm SEM: $77.13 \pm 7.13$ vs $68.13 \pm 6.96$, respectively, $\mathrm{p}<0.0001$ ) and in DLCO values (mean \pm SEM: $63.13 \pm 7.65$ vs 52.25 \pm 7.32 , respectively, $\mathrm{p}<0.001$ ) were observed. Moreover, a decrease of ground glass lesions was depicted in the analysis of HRCT of 5/8 patients. Despite the small numbers of patients and the concurrent administration of RTX with other immunosuppressing drugs (for instance MMF), the results are considered to be encouraging.

A promising open label study of Smith et al. [32] in patients with early diffuse scleroderma who received RTX for a 2-year period, showed a statistically but not clinically significant overall decrease of FVC, with a mean FVC $92.8 \%$ at baseline versus $84.7 \%$ at 24 months (MMA $\mathrm{p}=0.047$ ). DLCO also remained stable over the 2-years. Clinical insignificance was judged based on $<10 \%$ yearly change, which is arbitrary only.

Bosello et al. [33] administered RTX (RA scheme in 20 patients with early ( $<3$ years disease duration) and extensive scleroderma lung disease. By evaluating PFTs every 6 months and chest HRCT every 12 months they noticed stability of FVC, DLCO and chest HRCT lesions during the follow up period of 2 years. However, a marked heterogeneity was recorded for follow-up duration and for RTX cycles repetition patterns. It maybe noteworthy that among the patients enrolled, the most interesting patients with restrictive disease $(n=6)$ in PFTs had an increase of FVC but insignificant changes of DLCO and HRCT scores during the first year of RTX treatment. There were also
8 patients without restrictive disease in PFTs displaying no increases of the above parameters throughout the study.

In a retrospective analysis, Sharp et al. [34] studied 24 patients with resistant scleroderma lung disease, i.e. not responsive to previous immunosuppressive drugs. Administration of B cell depletion resulted in stabilization of FVC, DLCO and chest HRCT parameters. Lepri et al. [23] analyzed retrospectively patients with various ILD, including 23 patients with SSc-ILD. They were treated with RTX plus other DMARDs and were followed-up for 2-years. The mean dose of RTX was stated to be $2800 \mathrm{mg}$ during the $1 \mathrm{st}$ year and $1750 \mathrm{mg}$ during the $2 \mathrm{nd}$ year. In patients with SSc-ILD, FVC changed from $81.0 \%$ at baseline to $89.0 \%$ at 1 year $(\mathrm{p}=0.1)$ and 74.5 at 2 years $(\mathrm{p}=0.07)$. DLCO changes remained non-significant throughout. The results of this uncontrolled trial are confounded by the retrospective nature of the study and by the unclear RTX-dosing regimen.

Vilela et al. [35] described 10 patients with diffuse SSc that received RTX (RA scheme) and were evaluated 6 months later. There was no significant change in PFTs in all patients, neither in the subgroup with early disease (duration less than 4 years and refractory to standard treatment), nor in the subgroup with worsening, progressing pulmonary function. Sari et al. [36] claimed a beneficial effect of RTX therapy in 14 patients with SSc and chronic extensive lung involvement resistant to previous therapy. Although encouraging, the results are not comparable between patients because they received different numbers of cycles and different dosages of RTX. Uncontrolled trials are depicted in Table 2.

\section{$B$ cell depletion and safety issues}

B cell depletion treatment was well tolerated as the majority of the studies have reported. Quite a few patients may have reported gastrointestinal complaints; infusion reactions were sparsely mentioned. Infections represent our major concern and potential problem; respiratory tract infections were easily dealt with, as reported [27]. Taking into account that our patients are highly immunocompromised and have an extensive fibrous lung parenchyma, we have to weigh the pros and cons of initiating or not initiating B cell depleting therapy. Respiratory infections as an adverse event should not discourage us from administration RTX treatment; especially if the inflammatory process is decelerated by the drug as many trials point.

Table 1. Double controlled studies of SSc ILD treatment with RTX

\begin{tabular}{|c|c|c|c|c|c|}
\hline Author & No of patients & Disease type & RTX scheme & Follow up months & Outcome \\
\hline Daoussis, et al. 2010 & 8 & $\begin{array}{l}\text { SSc serious ILD RTX add on Tx vs } \\
\text { standard Tx }\end{array}$ & Lymphoma scheme & 12 & $\begin{array}{l}\text { Improvement of PFTS compared to } \\
\text { controls;steady HRCT lesions }\end{array}$ \\
\hline Jordan, et al. 2015 & 25 & SSc ILD RTX + stand vs standard Tx & RA scheme & 6 & Decline in FVC/stable DLCO \\
\hline Daoussis, et al. 2017 & 51 & SscILD RTX add on Tx Vs standard Tx & Lymphoma scheme & 48 & FVC increase/stable DLCO \\
\hline Boonstra M, et al. 2017 & 16 & SscILD & RA scheme & 24 & Stable PFTs and HRCT lesions \\
\hline Thiebeau, et al. 2018 & 13 & Progressive Ssc ILD & RA scheme & 24 & $\begin{array}{l}\text { No difference compared to control/ } \\
\text { improvement of PFTs in RTX group }\end{array}$ \\
\hline Sircar, et al. 2018 & 25 & SSc ILD RTXvs CYC & RA scheme & 6 & Improved FVC compared to CYC \\
\hline $\begin{array}{c}\text { M Elhai, M Boubaya, et al. } \\
2019 \text { EUSTAR }\end{array}$ & 146 patients & SSc ILD & RA scheme & 24 & Stable PFTs \\
\hline
\end{tabular}

Table 2. uncontrolled studies of SSc-ILD treatment with RTX

\begin{tabular}{|c|c|c|c|c|}
\hline Author & No of patients & Disease & RTX scheme & Follow up months \\
\hline Lafyatis, et al. 2009 & 15 & $\begin{array}{c}\text { Early SSc ILD RTX add on standard Tx not } \\
\text { progressive ILD }\end{array}$ & RA scheme & Stable PFTs no ILD progression \\
\hline Daoussis, et al. 2012 & 8 & SSc ILD RTX add on standard Tx & Lymphoma scheme & $\begin{array}{c}\text { Increase of FVC, DLCO, decrease } \\
\text { ground class }\end{array}$ \\
\hline Bosello, et al. 2015 & 20 & Early serious ILD SSc & RA scheme & 24 \\
\hline Sharp, et al. 2016 & 24 & Resistant SSc ILD to previous Tx & RA scheme & 24 \\
\hline Sari, et al. 2017 & 14 & Resistant SSc ILD & RA scheme & 6 \\
\hline
\end{tabular}




\section{Conclusion}

The progressive, quality-of-life decreasing and life-threatening nature of SSc-ILD represent a major challenge to identify and apply an aggressive and effective treatment yet with an acceptable safety profile, that will at least slow down disease progression. Apart from the current standard-of-care treatment with MMF, B cell depletion appears to be beneficial and acceptably safe for scleroderma lung disease and the 2 different treatment approaches can be combined. A small number of case reports and more importantly a good number of open-label studies are in agreement with the above statement. Both controlled and uncontrolled studies of using treatment with RTX are encouraging. Based on data discussed herein, patients with SSc and either early- or long-standing disease with significant ILD that is resistant to previous therapy may represent successful candidates for B cell depletion treatment. In addition, despite a semi-quantitative approach, imaging studies may also depict improvements following RTX treatment. B cell depletion administered in repeated cycles may be a better approach in contrast to a single infusion.

Published trials suffer from a marked multifactorial heterogeneity: differences in disease duration, previous and current treatments, extent of lung disease, lack of clearly defined cut-off values for PFT parameters such as the FVC and DLCO, as well a non-digital scoring system for HRCT lesions, all limit our clear understanding of the benefits expected from B cell depletion treatment in patients with SSc-ILD. A welldesigned, large enough study is needed to overcome such limitations. Employing B cell depletion as a first-line treatment is also another question waiting to be addressed. Despite all the above limitations and downsides, one should keep in mind that the vast majority of studies published so far support a significant improvement of SSc-ILD in patients treated with a B cell depleting therapeutic approach.

\section{References}

1. Varga J, Trojanowska M, Kuwana M (2017) Pathogenesis of systemic sclerosis: Recent insights of molecular and cellular mechanisms and therapeutic opportunities. Journal of Scleroderma and Related Disorders 2: 32.

2. Moore OA (2013) Extent of disease on high-resolution computed tomography lung is a predictor of decline and mortality in systemic sclerosis-related interstitial lung disease. Rheumatol (United Kingdom) 52: 155-160. [Crossref]

3. Goh NS (2017) Short-Term Pulmonary Function Trends Are Predictive of Mortality in Interstitial Lung Disease Associated with Systemic Sclerosis. Arthritis Rheumatol 69: 1670-1678. [Crossref]

4. Daoussis D (2012) B-cell depletion therapy in patients with diffuse systemic sclerosis associates with a significant decrease in PDGFR expression and activation in spindlelike cells in the skin. Arthritis Res Ther 14: R14. [Crossref]

5. Daoussis D, Liossis SNC (2013) B cells tell scleroderma fibroblasts to produce collagen. Arthritis Research and Therapy 15: 125.

6. Distler O, Volkmann ER, Hoffmann-Vold AM, Maher TM (2019) Current and future perspectives on management of systemic sclerosis-associated interstitial lung disease. Expert Review of Clinical Immunology 15: 1009-1017.

7. Kowal-Bielecka O (2017) Update of EULAR recommendations for the treatment of systemic sclerosis. Ann Rheum Dis. [Crossref]

8. DaoussisD, Liossis SN, Kalogeropoulou C, Kazantzi A, Yiannopoulos G, et al. (2010) Improvement of lung function and skin thickening following long term rituximab treatment in patients with scleroderma. Clin Exp Rheumatol 30: S17-S22 [Crossref]

9. Daoussis D (2012) Effect of long-term treatment with rituximab on pulmonary function and skin fibrosis in patients with diffuse systemic sclerosis. Clin Exp Rheumatol 30: S17-S22. [Crossref]

10. Daoussis D (2010) Is There a Role for B-cell Depletion as Therapy for Scleroderma? A Case Report and Review of the Literature. Semin Arthritis Rheum 40: 127-136. [Crossref]

11. Daoussis D (2010) Evidence of skin remodelling and improvement of lung function following 2 years of rituxi-mab treatment in a patient with diffuse scleroderma. Clin Exp Rheumatol 30: S17-S22. [Crossref]
12. Moazedi-Fuerst FC (2014) Rituximab for systemic sclerosis: arrest of pulmonary disease progression in five cases. Results of a lower dosage and shorter interval regimen. Scandinavian Journal of Rheumatology. [Crossref]

13. Sakkas LI, Bogdanos DP (2016) Systemic sclerosis: New evidence re-enforces the role of B cells. Autoimmunity Reviews. [Crossref]

14. Daoussis D, Andonopoulos AP (2011) Rituximab in the treatment of systemic sclerosisassociated interstitial lung disease: Comment on the article by Yoo. Rheumatol Int 31: 841-842 [Crossref]

15. Lafyatis R, Hara OC, Feghali-Bostwick CA, Matteson E (2007) B cell infiltration in systemic sclerosis-associated interstitial lung disease. Arthritis Rheum 56: 3167-3168. [Crossref]

16. Saito S, Kasturi K, Bona C (1999) Genetic and immunologic features associated with scleroderma-like syndrome of TSK mice. Current Rheumatology Reports 1: 34-37. [Crossref]

17. Asano N (2004) B lymphocyte signalling established by the CD19/CD22 loop regulates autoimmunity in the tight-skin mouse. Am J Pathol 165: 641-650. [Crossref]

18. Hasegawa M (2006) B-lymphocyte depletion reduces skin fibrosis and autoimmunity in the tight-skin mouse model for systemic sclerosis. Am J Pathol 169: 954-966. [Crossref]

19. Le Huu D (2013) Donor-derived regulatory B cells are important for suppression of murine sclerodermatous chronic graft-versus-host disease. Blood 121: 3274-3283. [Crossref]

20. Komura K (2008) CD19 regulates the development of bleomycin-induced pulmonary fibrosis in a mouse model. Arthritis Rheum 58: 3574-3584. [Crossref]

21. Allen JL (2012) B cells from patients with chronic GVHD are activated and primed for survival via BAFF-mediated pathways. Blood 120: 2529-2536. [Crossref]

22. Daoussis D (2010) Experience with rituximab in scleroderma: Results from a 1-year, proof-of-principle study. Rheumatology 49: 271-280. [Crossref]

23. Lepri $\mathrm{G}$ (2016) Effects of rituximab in connective tissue disorders related interstitial lung disease. Clin. Exp. Rheumatol 100: 181-185. [Crossref]

24. Daoussis D (2017) A multicentre, open-label, comparative study of B-cell depletion therapy with Rituximab for systemic sclerosis-associated interstitial lung disease. Semin Arthritis Rheum 46: 625-63. [Crossref]

25. Boonstra M (2017) Rituximab in early systemic sclerosis. RMD Open 98: e17110. [Crossref]

26. Thiebaut M (2018) Efficacy and safety of rituximab in systemic sclerosis: French retrospective study and literature review. Autoimmunity Reviews 17: 582-587. [Crossref]

27. Elhai M (2019) Outcomes of patients with systemic sclerosis treated with rituximab in contemporary practice: A prospective cohort study. Ann Rheum Dis 78: 979-987. [Crossref]

28. Sircar G (2018) Intravenous cyclophosphamide vs rituximab for the treatment of early diffuse scleroderma lung disease: Open label, randomized, controlled trial. Rheumatol. (United Kingdom), 2018.

29. Saunders P (2017) Rituximab versus cyclophosphamide for the treatment of connective tissue disease associated interstitial lung disease (RECITAL): Study protocol for a randomised controlled trial. Trials 23: 411-415. [Crossref]

30. Ebata S (2019) Rituximab therapy is more effective than cyclophosphamide therapy for Japanese patients with anti-topoisomerase I-positive systemic sclerosis-associated interstitial lung disease. J Dermatol 46: 1006-1013. [Crossref]

31. Lafyatis R (2009) B cell depletion with rituximab in patients with diffuse cutaneous systemic sclerosis. Arthritis Rheum 60: 578-583. [Crossref]

32. Smith V (2013) Two-year results of an open pilot study of a 2-treatment course with rituximab in patients with early systemic sclerosis with diffuse skin involvement. $J$ Rheumatol 40: 52-57. [Crossref]

33. Bosello SL (2015) Long-term efficacy of B cell depletion therapy on lung and skin involvement in diffuse systemic sclerosis. Semin Arthritis Rheum 44: 428-436.

34. Sharp C (2016) Rituximab in autoimmune connective tissue disease-associated interstitial lung disease. Rheumatol. (United Kingdom).

35. Vilela VS (2016) Rituximab for the therapy of systemic sclerosis: a series of 10 cases in a single center. Rev Bras Reumatol 56: 458-463. [Crossref]

36. Sari A (2017) Rituximab experience in patients with long-standing systemic sclerosisassociated interstitial lung disease: A series of 14 patients. J Clin Rheumatol 23: 411415. [Crossref]

Copyright: (C2020 Bounia CA. This is an open-access article distributed under the terms of the Creative Commons Attribution License, which permits unrestricted use, distribution, and reproduction in any medium, provided the original author and source are credited. 